\title{
A Workflow Model based on Homogeneous Activities"
}

\author{
F. Yang ${ }^{1}$, Y. Z. Zhu ${ }^{2}$ and Y. Luo ${ }^{1}$ \\ ${ }^{1}$ Beijing Institute Petrochemical Technology, China \\ ${ }^{2}$ Beihang University, China \\ yangfei-wu@sohu.com
}

\begin{abstract}
In process-oriented software systems, many processes have large number of parallel redundant activities which are often used in split and merge workflow structures and make workflow model too complex to manage. Aiming at the homogeneity of redundant activities the structure characteristics of these homogeneous activities are analyzed and they are being classified into eight modes. Also the constraints is identified which defined the coexistence of these modes. At last a workflow model based on homogeneous activities is presented. Comparison shows that the method has obvious advantage in reducing description time and maintaining cost of workflow model.
\end{abstract}

Keywords: Workflow, Homogeneous Activity, Redundant Activities, Structure Mode, Constraints

\section{Introduction}

In the cross-regional distributed large enterprise business processes, there usually exists complex business logic composed of split and merge. A large number of duplicate activity information exist in those modes, including task to performed, data used and precursor/subsequent structure, etc. [1,2]. These activities have the same position, goal and data which are called redundant activities in workflow model. When the number of redundant activities is large, using traditional workflow modeling method not only takes much time to describe activities, but also generates a huge processes difficult to maintain [3]. Redundant activities is the difficulty of workflow research area and it is included into workflow patterns involving multiinstance mode [4-5]. But multi-instance mode includes a large range and can't solve redundant activities in different scenarios, let alone the distributed workflow management system [6].

\section{Related Work}

In recent works on distributed workflow management systems, Muthusamy [7-8] developed a flexible and distributed platform to develop, execute and monitor business process supporting service discovery and composition which can describe redundant activities but the description method is too complex for workflow modeler.

Khalaf [9] presented a BPEL fragmentation covering data and explicit control dependencies to handle fragmenting loops. Hamann [10] proposed a migration data meta-model to realize the runtime migration of business processes, which improved the flexibility of the distribution of the ad-hoc workflow. The above workflow models are extended from the BPEL which can't describe redundant activities in simple forms.

\footnotetext{
* Supported by the Project of Excellent Talents Cultivation in Beijing(2013D005005000003) and National Undergraduate Training Programs for Innovation and Entrepreneurship(2014X00022)
} 
Besides, most workflow management systems such as Staffware, WebSphere, FLOWer and COSA, are able to support part multiple-instances patterns through some special mechanisms, such as "bundle model" or "dynamic parallel process management table"[11-12]. However, their description methods are too complex to support complicated workflow model containing multi-level split-merge structures, also most of them can be used only in centralized environment.

Our work distinguishes itself from other methods by concentrating on a small part of multiple-instances modes -- homogeneous activities. Also we propose workflow model based on homogeneous activities to reduce the complexity of modeling.

\section{Homogeneous Activity Mode}

Homogenous activity mode is a kind of workflow pattern in which a set of redundant activities can be represented by a special activity called homogenous activity. Homogenous activity mode is divided into Static Structure mode and Dynamic Structure mode, the former focuses on describing the topology relationships between redundant activities and the latter describes how to dynamically select running nodes.

\subsection{Static Structure Mode}

Homogeneous activity mode can be divided into starting mode, serial mode, parallel mode and sub-flow model.

\section{Starting Mode}

When homogeneous activity acts as a workflow starting node, the pattern is called starting mode. Here, redundant activities represented by homogeneous activity have a parallel XOR relationship shown in Figure 1. They also have permission to start workflow instance, but only one redundant activity is actually executed in one workflow instance. Multiple possible starting branches existing in homogeneous activity can significantly reduce the size of process, though not allowed in many workflow management system it is very useful.

Redundant activities

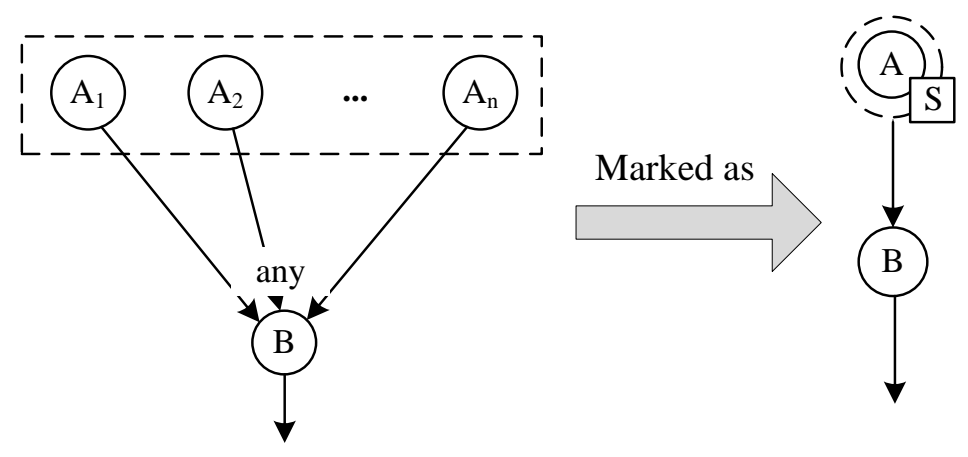

Figure 1. Starting Mode

\section{Serial Mode}

When homogeneous activity has a relationship of sequential, the pattern is called serial mode. Redundant activities are organized in the follow three ways shown in Figure 2 in serial mode. 

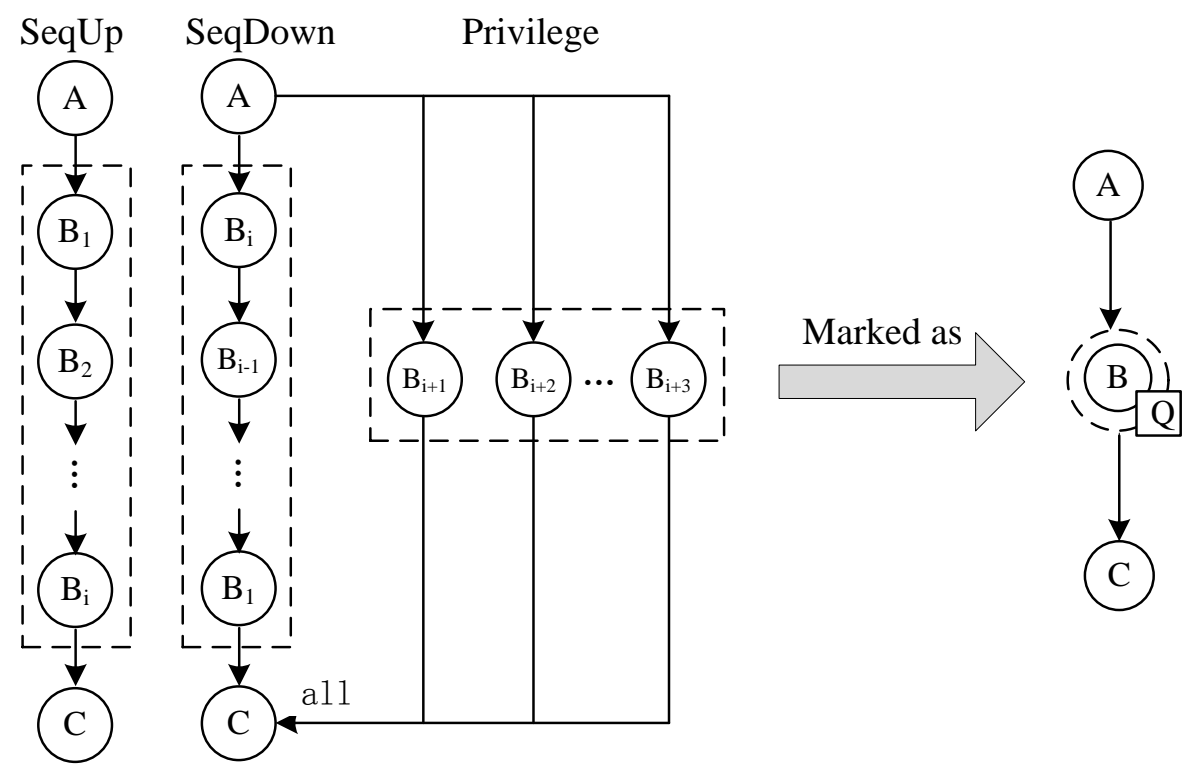

Figure 2. Serial Mode

Sequence Up(SeqUp). Redundant activities are executed sequentially from low to high according to performer position. It is common in approval processes to realize approval step by step.

Sequence Down(SeqDown). Redundant activities are executed sequentially from high to low according to performer position. It is often used in dissemination processes to achieve progressively sign.

Privilege. Homogeneous activity exists with serial activities simultaneously, but in dependent of serial activities. privilege mode can be used if some performers are unable to handle workflow instance because of special reasons in SeqUp or SeqDown mode. For example, some high position leaders often can't issue documents due to time constraints, in order to prevent delay in process execution, privilege mode is used to allow them to retroactive sign while others issue documents with normal procedure. Privilege mode has often been used without it many workflow will not be processed timely.

\section{Parallel Mode}
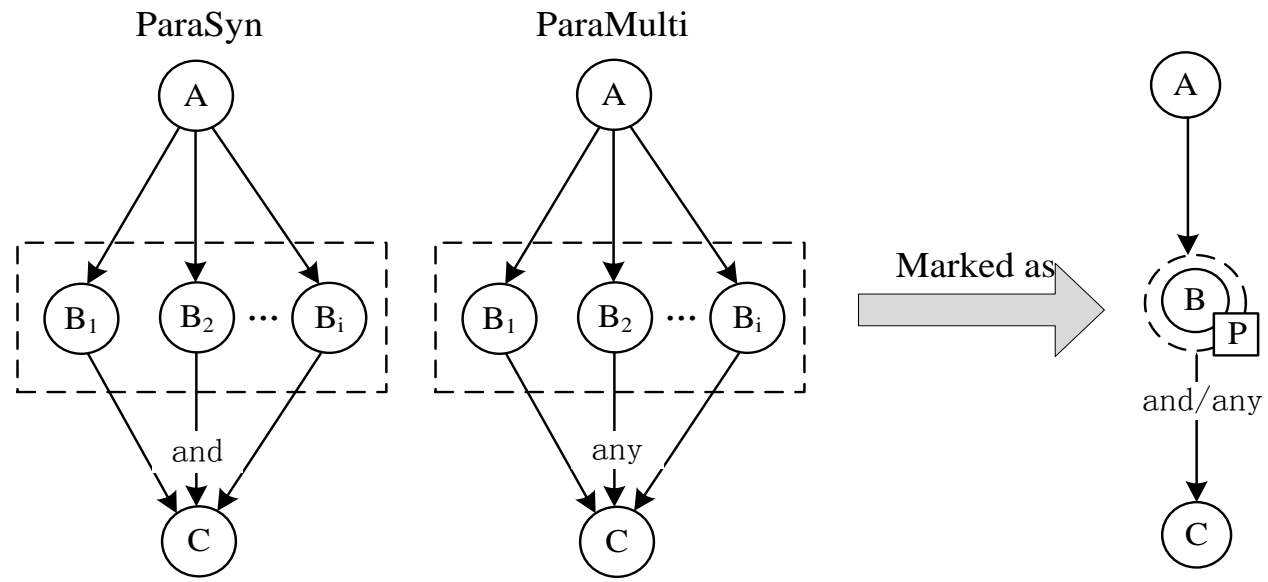

Figure 3. Parallel Mode 
When homogeneous activity has a relationship of concurrent, the pattern is called parallel mode. Parallel mode includes two types shown in Figure 3.

Parallel Synchronous merge (ParaSyn). Activity can be performed only after all precursors have been completed. ParaSyn is essentially a multi-node cooperative mode.

Parallel Multiple merge (ParaMulti). Activity can be performed once there has a precursor completed. It can be used to increase workflow instance number.

\section{Sub-flow Mode}

When homogeneous activity is composed by sub workflow, the pattern is called subflow mode shown in Figure 4. Sub-flow mode can be realized nesting without depth limit thus to support more complex workflow model.

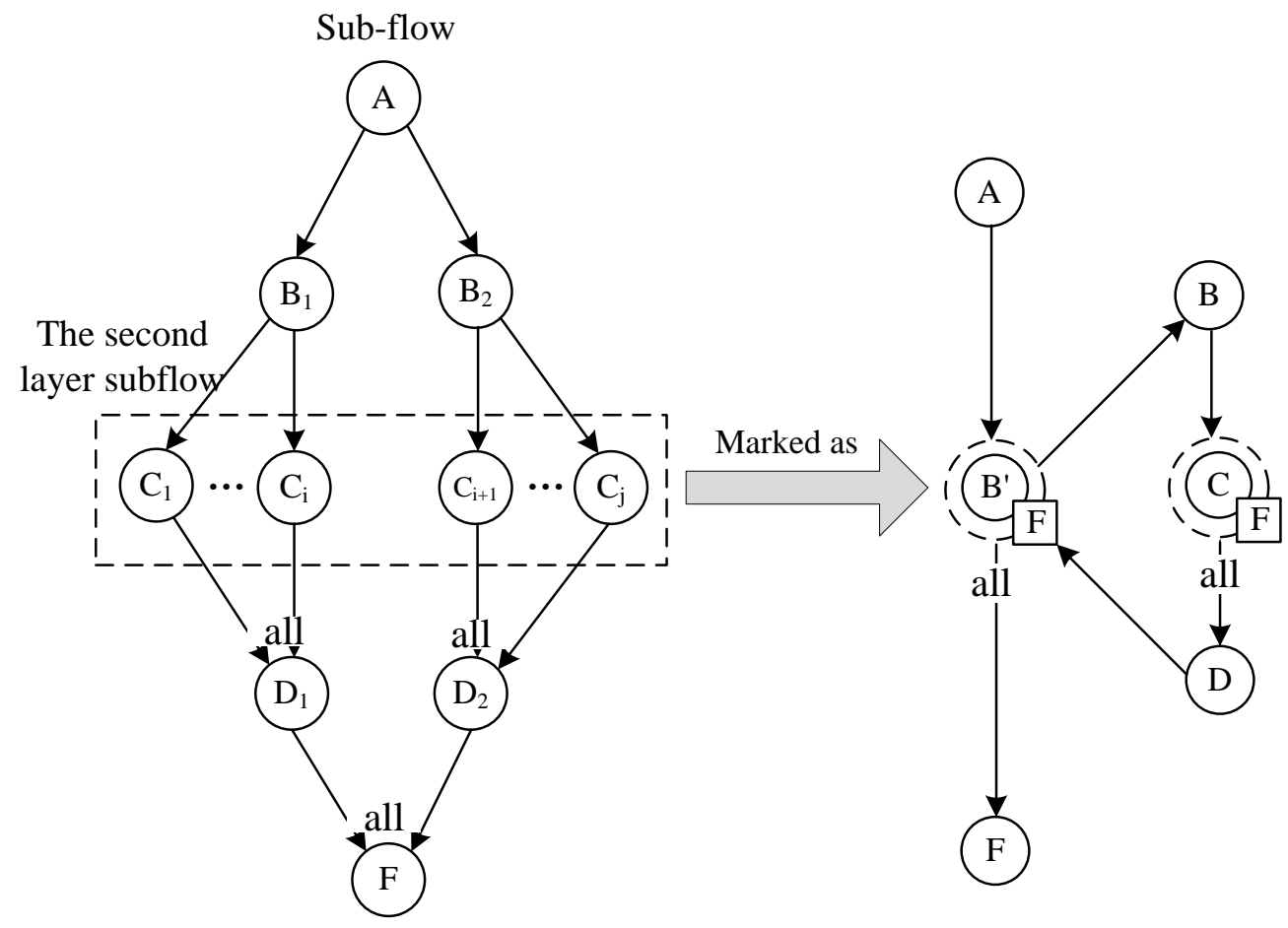

Figure 4. Sub-flow Mode

\subsection{Dynamic Structure Mode}

Dynamic Structure mode of homogeneous activity usually relies on its precursor because it determines which redundant activities can be executed. Dynamic Structure mode includes auto selection mode, manual selection mode, association selection mode and association avoidance mode.

\section{Auto Selection Mode}

The precursor of homogeneous activity automatically determines whether redundant activities are executed, the pattern is called auto selection mode. It is generally used for one choice, namely only one activity can be automatically selected to be executed. Selection algorithm such as RoundRobin, Random and Weight shown in Figure 5 can be used. Auto selection mode is used to automatically distribute tasks. For example, when several performers undertake tasks from the same queue, their property value can be set to ensure the workload balance. 


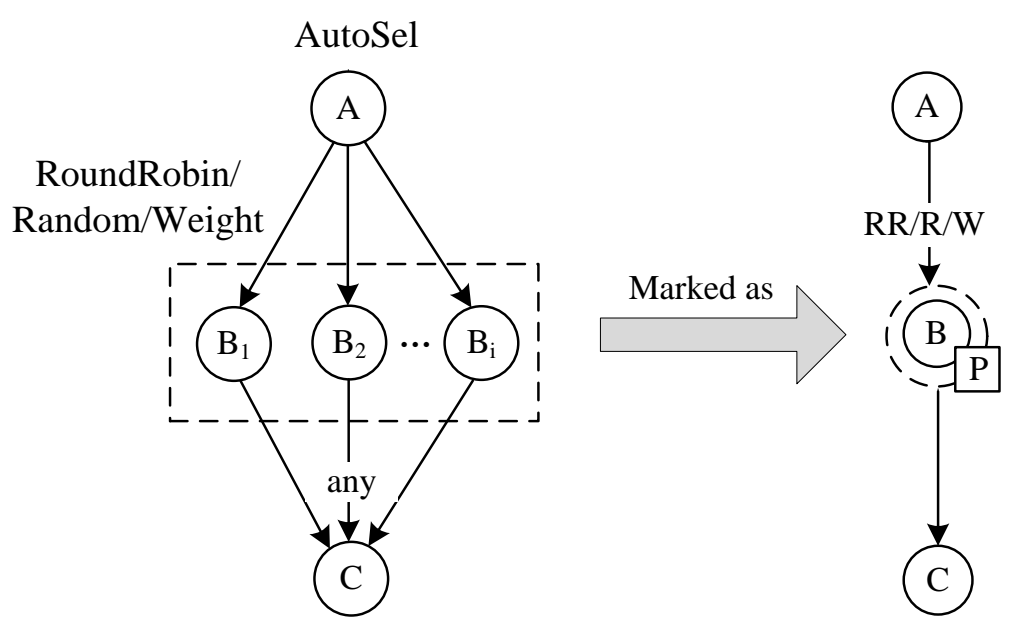

Figure 5. Auto Selection Mode

\section{Manual Selection Mode}

Manual selection mode can be divided into two kinds of single selection and multiple selection shown in Figure 6. The former is relatively simple and equivalent to the combination of XOR-split and XOR-merge, while the latter is more complex and can be used with several other modes simultaneously. Manual selection mode provides sufficient flexibility for instances and improves the representation ability of workflow model.

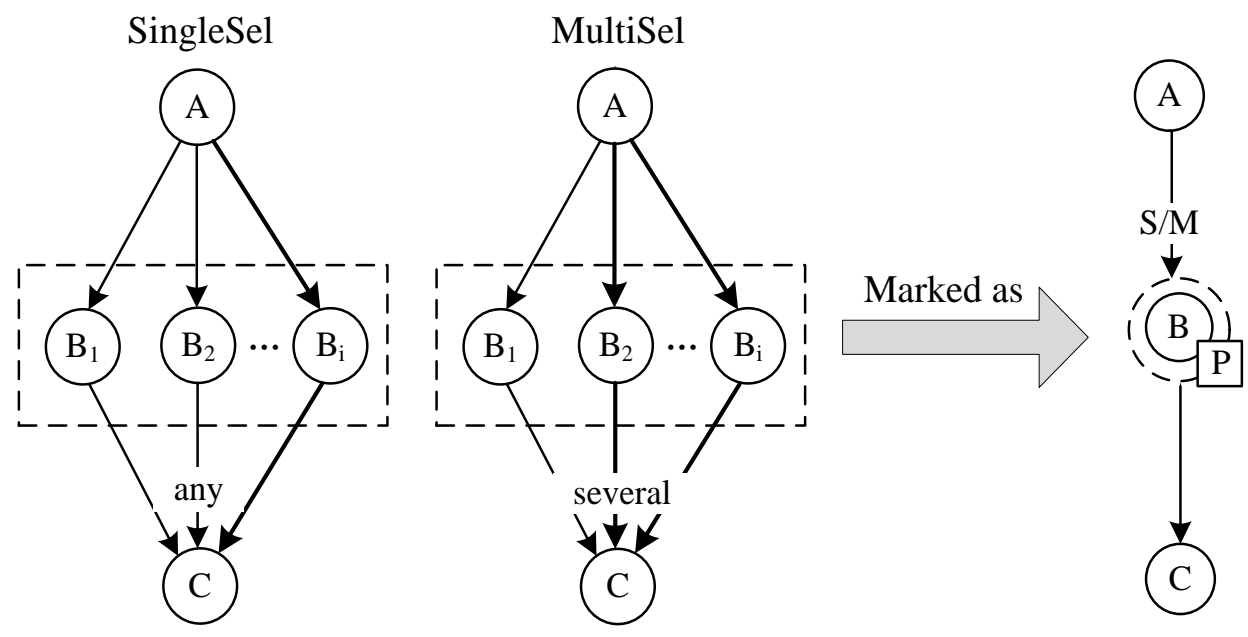

Figure 6. Manual Selection Mode

\section{Association Selection Mode}

When the performer of homogeneous activity has some special relationship with its precursor performer, the pattern is called association selection mode. It is a common and useful selection mode to describe "relative role" of performer and complex structure combined with sub-flow mode shown in Figure 7. Association selection mode is a homogeneous activity mode which can be used with auto selection mode and manual selection mode, also can be used with serial mode and parallel mode. 


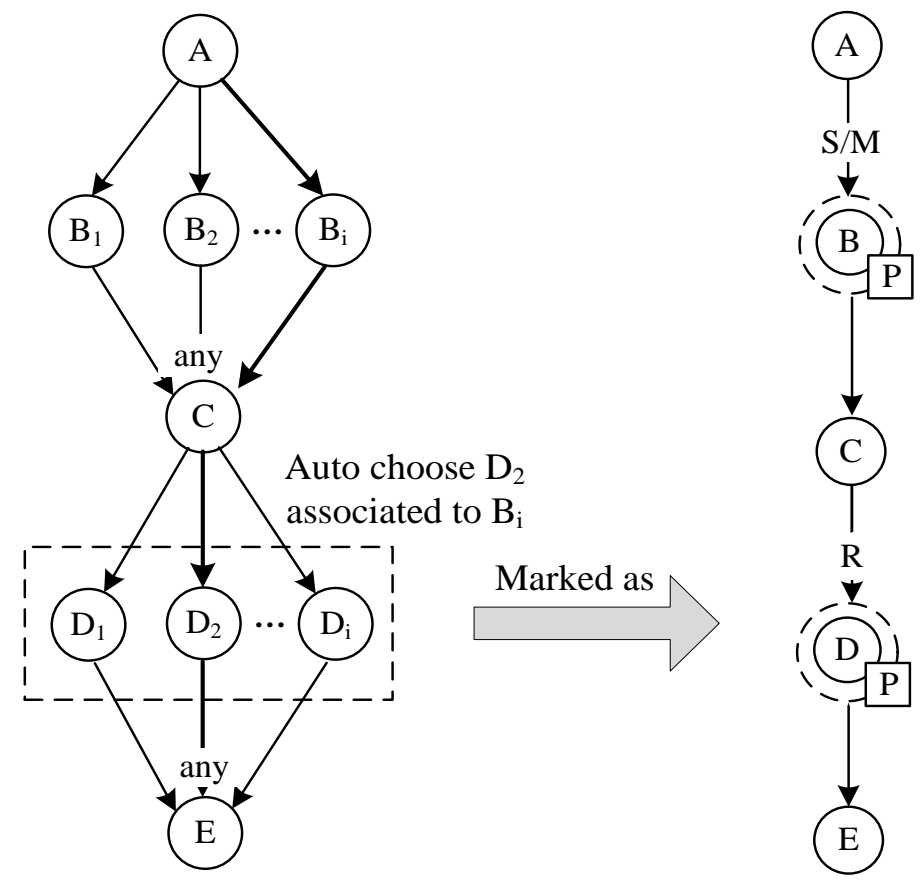

Figure 7. Association Selection Mode

\section{Association Avoidance Mode}

When the performer of homogeneous activity can't have some relationships with its precursor performer, the pattern is called association avoidance mode. In converse to association selection mode, it is used to avoid some options, commonly activity performer. For example, if the performer or its organization process the precursor activity, then he/she is not allowed to process the current activity. Association avoidance mode has a very wide range of uses and can be combined with most other homogeneous activity mode.
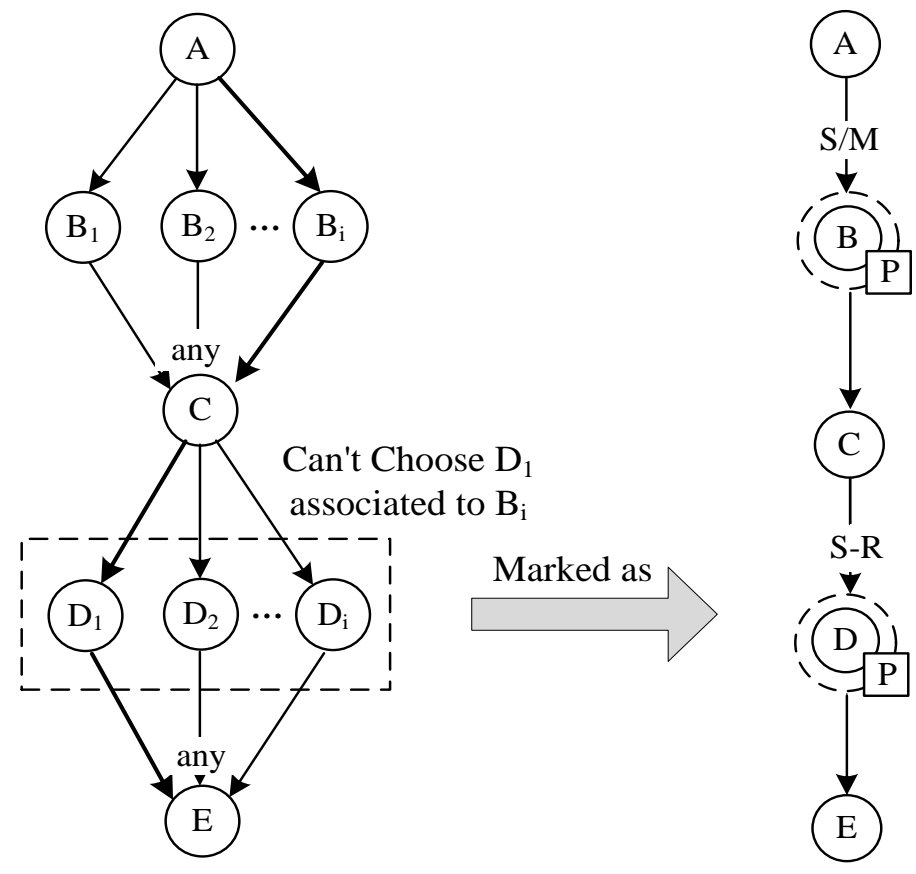

Figure 8. Association Avoidance Mode 


\subsection{Homogeneous Activity Mode Constraints}

When configuring homogeneous activity, the mode relationships of redundant activities must be known except the set of redundant activities. The following constraint rules can be concluded from the relationships of the above eight types.

(1) Serial mode and parallel mode can't coexist.

(2) Manual selection mode and auto selection mode can't coexist.

(3) Serial mode can coexist with auto selection mode and sub-flow mode.

(4) Starting mode must be parallel mode and can't be serial mode. Also it can only coexist with sub-flow mode and can't coexist with selection mode because of no precursor.

(5) Association selection mode and association avoidance cant' coexist with starting mode and serial mode.

(6) Sub-flow mode can coexist with any other mode.

The constraint of homogeneous activity modes are shown in Figure 9. Where the solid line represents both ends of the connection can coexist and rectangle represents the set of modes.

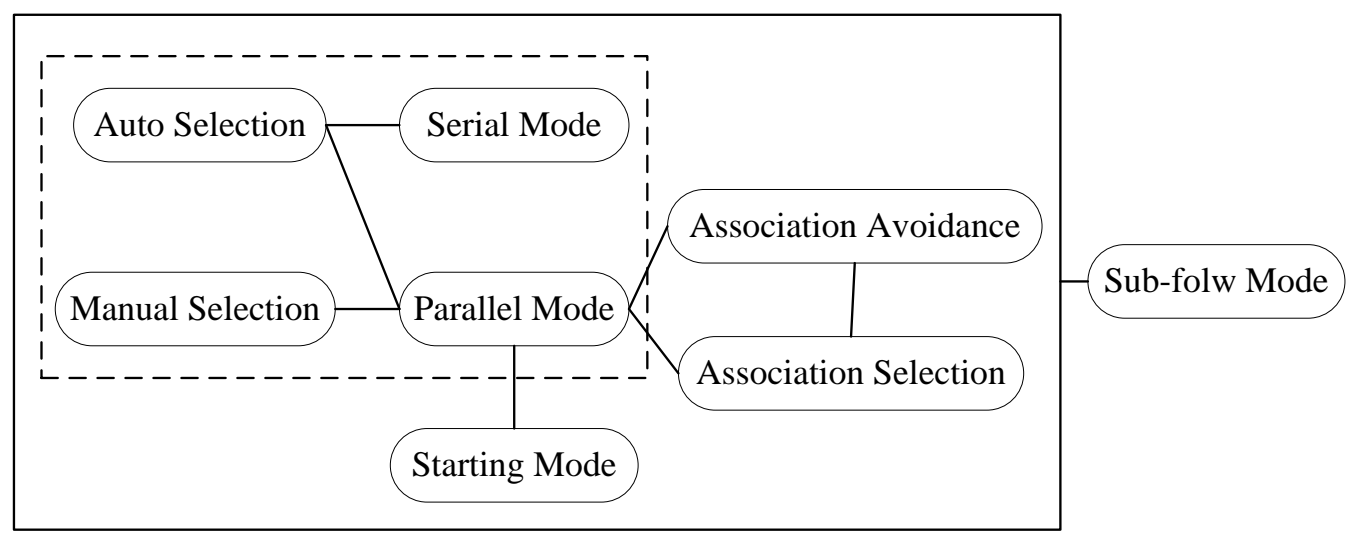

Figure 9. Homogeneous Activity Mode Constraints

\section{Workflow Mode based on Homogeneous Activity}

Use footnotes sparingly (or not at all!) and place them at the bottom of the column on the page on which they are referenced. Use Times New Roman 9-point type, singlespaced with 10-point interlining spacing. To help your readers, avoid using footnotes altogether and include necessary peripheral observations in the text (within parentheses, if you prefer, as in this sentence).

\subsection{Homogeneous Activity Workflow Mode}

Homogeneous activity workflow model $h w f=(h w i d, A C T, H A C T, F C, W F D)$.

(1) $h w i d$ is the identification of homogeneous activity workflow model.

(2) $A C T=\left\{a c t_{1}, a c t_{2}, \cdots, a c t_{n}\right\}$ is the collection of basic activities ${ }^{[13,14]}$.

(3) HACT $=\left\{\right.$ hact $_{1}$, hact $_{2}, \cdots$, hact $\left._{n}\right\}$ is the collection of homogeneous activities.

(4) $F C=($ link, forwardCond $)$ is inter-active transition conditions in which forwardCond is transfer condition expression, link $\in(A C T \cup H A C T) \times(A C T \cup H A C T)$.

(5) WFD is the collection of workflow data. 


\subsection{Homogeneous Activity}

hact $=($ hactid, Role, SRole, smode, dmode, $C D$, subhwf $)$ is Homogeneous activity.

(1) hactid is the identification of homogeneous activity.

(2) Role $=\left(\right.$ role $_{1}$, role $_{2}, \cdots$, role $\left._{n}\right)$ is the collection of performers consistent with association mode constraints. Association mode includes association selection mode and association avoidance mode. For each performer role $\forall$ role $\in$ Role , role $\in((U N \cup$ thisUnit $) \times R) \cup$ thisUnit, which $U N$ is the collection of organization units, $R$ is the collection of roles in organization unit, thisUnit is an special organization unit to indicates the node of head department, usually used in sub workflow to describe the relative role.

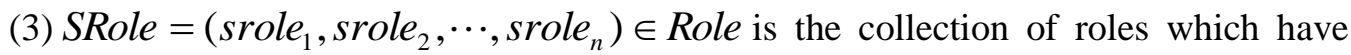
privileged. When serial mode is used, namely smode $\in\{$ SeqDown, SeqUp $\}$, privilege role can work.

(4) smode $\in\{$ SeqDown, SeqUp, ParaSyn, ParaMulti $\}$ is the collection of static structure mode of homogeneous activity.

(5) dmode $\in\{$ RoundRobin,Random,Weight, SingleSel,MultipleSel $\}$ is the collection of dynamic structure mode of homogeneous activity.

(6) $C D \subset W F D$ is data set concerned with the activity.

(7) subhwf is sub workflow model with the same property of $h w f$.

\section{Case Study}

A purchasing process of a large enterprise is shown in Figure 10 which is related to six department including financial department(FD), purchase department(PD), ERP department(ED), BI department(BD), E-business department(EBD) and Administration department(AD). ED, BD, EBD consist of three separate project teams and PD consists of two procurement centers.

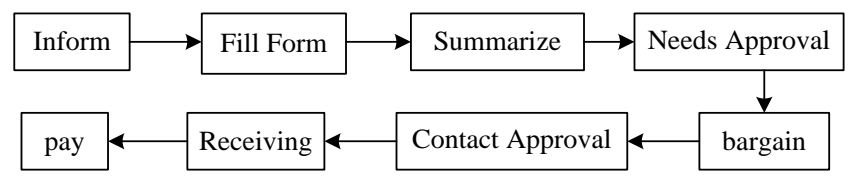

Figure 10. Purchasing Process

The purchasing process is started by the managers of ED, BD and EBD, and the purchasing instructions are forwarded to each project team in his organization. After all material requirement forms are received by the manager they can be collected to generate purchase requisitions and submit headquarter. Once reviewed the requisitions will be distributed to the corresponding procurement center according to material type. After bargaining the result will be handed over the headquarter for contact. Only material has been received, FD can pay in accordance with contract. Then the purchasing process is completed.

The homogeneous activity workflow model of purchasing process is shown in Error! Reference source not found.(a). Homogeneous activity $\operatorname{san}_{1}$ is responsible for the department purchase requisition consisted with starting mode which corresponds to the sub workflow of the collection of procurement requirements. Activities $a n_{11}$ and $a n_{13}$ executed by project managers realize to dispatch and collect work of material requisition 
forms. The activity $\operatorname{san}_{12}$ is the homogeneous activity of sub workflow consisted with ParaSyn mode to be executed by each project to realize form filling work.

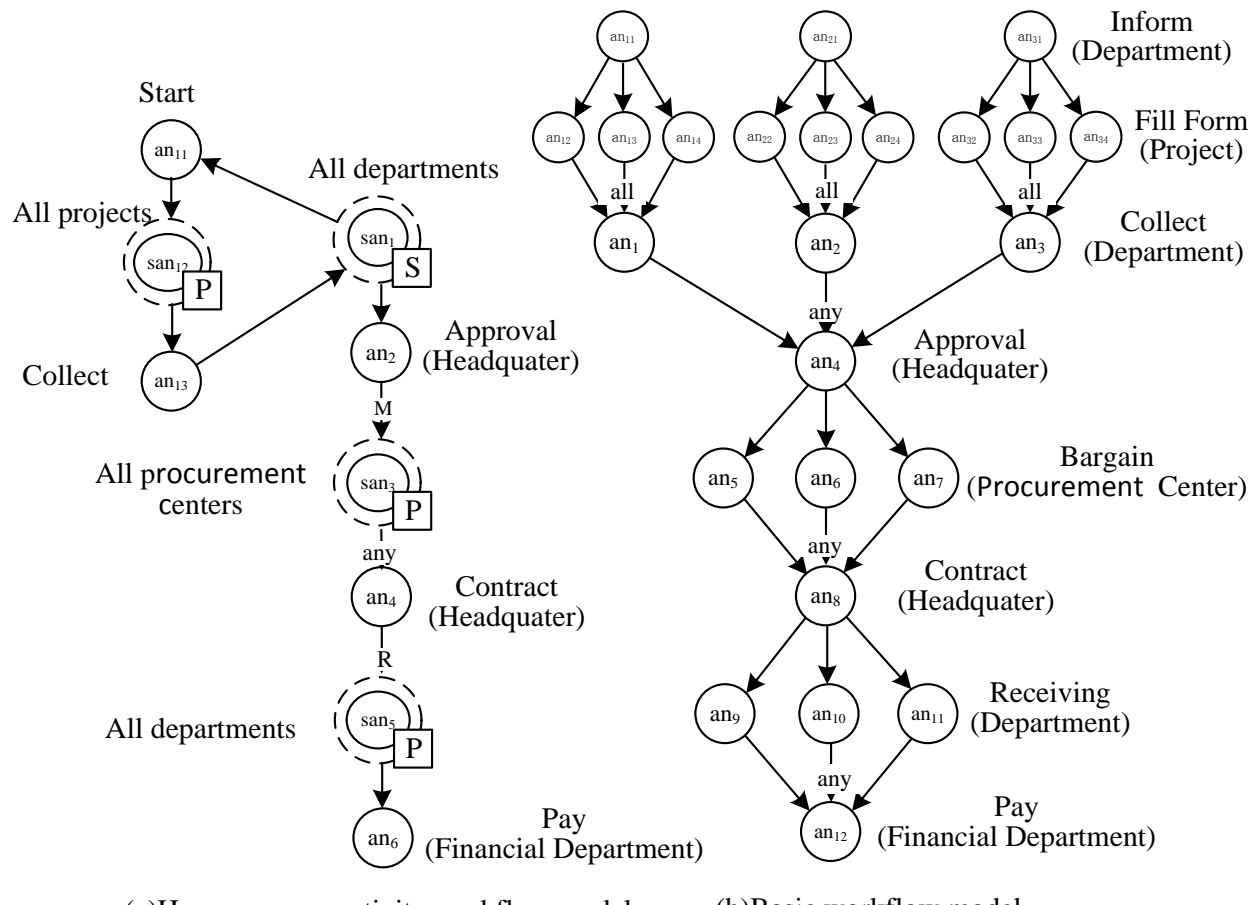

(a)Homogeneous activity workflow model

(b)Basic workflow model

\section{Figure 11. Workflow Model Diagram}

The purchasing request proposed by $s a n_{1}$ is forwarded to activity $a n_{2}$ which represents the approval of headquarters. Any workflow instance coming from $\operatorname{san}_{1}$ can activate $a n_{2}$. The corresponding procurement center will be selected based on material type in activity $a n_{2}$.Activity $\operatorname{san}_{3}$ consisted with auto selection and ParaMulti mode realizes the work of bargaining. After suppliers are determined the contract will be send to headquarters. Activity $a n_{4}$ realizes the approval of contract of headquarters. Multiple workflow instances are generated after activity $a n_{4}$ because every procurement center will submit his own contract and each contract needs an instance to be executed. Also $a n_{4}$ will notify activity $s a n_{5}$ to receive which consisted with association selection mode. Once all materials are received by $\mathrm{ED}$ or $\mathrm{BD}$ or EBD, the department will execute activity $a n_{6}$ to inform FD to pay, then the instance is being completed.

The basic workflow model is shown in Figure 11(b). From the comparison of Figure 11(a) and (b), we can see the introduction of homogeneous activity greatly simplifies the complexity of workflow model.

\section{Comparison}

The distributed workflow management system EasyWork[15] developed based on homogeneous activity is compared with other mainstream workflow management system including Staffware, COSA, FLOWer, WebSphere MQ Workflow and SAP/R3. The result can be seen in Table 1 . Also fully support is represented by " $\sqrt{ }$ ", not support is represented by " $x$ ”, part support is represented by “-”. Some parts of information comes in the table is derived from $[12,16]$. 
Table 1. Comparison of Homogeneous Activity Describing

\begin{tabular}{l|c|c|c|c|c|c|c|c}
\hline WFS & $\begin{array}{c}\text { Star } \\
\text { t-ing }\end{array}$ & $\begin{array}{l}\text { Seria } \\
\text { l }\end{array}$ & $\begin{array}{c}\text { Para } \\
\text {-llel }\end{array}$ & $\begin{array}{c}\text { Auto- } \\
\text { Sel }\end{array}$ & $\begin{array}{c}\text { Single- } \\
\text { Sel }\end{array}$ & $\begin{array}{c}\text { Multi- } \\
\text { Sel }\end{array}$ & $\begin{array}{c}\text { Asso } \\
\text {-Sel }\end{array}$ & $\begin{array}{c}\text { Asso- } \\
\text { Avoid }\end{array}$ \\
\hline Staffware 9 & & $\times$ & $\times$ & $\times$ & $\sqrt{ }$ & $\times$ & $\times$ & $\times$ \\
\hline COSA 4.2 & & $\times$ & $\times$ & $\sqrt{ }$ & $\sqrt{ }$ & $\times$ & $\sqrt{ }$ & $\times$ \\
\hline FLOWer 3 & & $\times$ & $\times$ & $\times$ & $\sqrt{ }$ & $\times$ & $\sqrt{ }$ & $\times$ \\
\hline Meteor & & $\times$ & $\times$ & $\times$ & $\times$ & $\times$ & $\times$ & $\times$ \\
\hline $\begin{array}{l}\text { WebSphere } \\
\text { MQ }\end{array}$ & & $\sqrt{ }$ & - & $\times$ & $\sqrt{ }$ & & & $\sqrt{ }$ \\
Workflow & & & & & & & & \\
3.3.4 & & $\sqrt{ }$ & $\times$ & $\times$ & $\sqrt{ }$ & $\times$ & $\sqrt{ }$ & $\times$ \\
\hline SAP R3 & & $\sqrt{ }$ & $\sqrt{ }$ & $\sqrt{ }$ & $\sqrt{ }$ & $\sqrt{ }$ & $\sqrt{ }$ & $\sqrt{ }$ \\
\hline EasyWork & $\sqrt{ }$ & & & & &
\end{tabular}

It can be seen from the table that WebSphere MQ Workflow can support homogeneous activity to a certain extent and SAP R3 provides only limited support. In contrast EasyWork has the unique advantage.

\section{Conclusions}

In this research, the concept of homogeneous activity is presented according to the homogeneity of redundant activities. Then eight workflow modes are summarized based on the characteristic of homogeneous activities including starting mode, serial mode, parallel mode, sub-flow mode, auto selection mode, manual selection mode, association mode and association avoidance mode. After analyzing the constraints of the above modes the workflow model based on homogeneous activities is proposed which can not only significantly improve the description ability but also can reduce the maintenance cost.

\section{References}

[1] W. M. P. van der Aalst and M. Weske, "Seminal Contributions to Information Systems Engineering", (2013)

[2] D. Fahland and W.M.P. van der Aalst, "Information Systems", 38, 4(2013)

[3] W.M.P. van der Aalst, "ISRN Software Engineering", (2013)

[4] A.P. Barros and A. Grosskopf, "Multiple instance management for workflow process models", U.S. Patent 8,424,011, Apr 16 (2013)

[5] N. Russell, A.H.M. ter Hofstede and N. Mulyar, BPM Center Report BPM-06-22, (2006)

[6] W.M.P. van der Aalst, A.H.M. ter Hofstede, B. Kiepuszewski and Alistair P. Barros, "Distributed and Parallel Databases", 14, 1(2003)

[7] V. Muthusamy, "Flexible distributed business process management", University of Toronto, 2012.

[8] S. Ganesan, Y. Yoon and H. Jacobsen, "NIÑOS take five: the management infrastructure for distributed event-driven workflows', Proceedings of the Fifth ACM International Conference on Distributed EventBased Systems, (2011) July 11-15; New York, USA

[9] R. Khalaf and F. Leymann, "Information Systems", 37, 6 (2012)

[10] K. Hamann, S. Zaplata and W. Lamersdof, "Software Services and Systems", 1, 3(2010)

[11] E. Deelman, D. Gannon, M. Shields and I. Taylor, "Future Generation Computer Systems", 25, 5(2009)

[12] N. Russell, A. ter Hofstede, D. Edmond and W. M. P. van der Aalst, "Proceedings of 24th International Conference on Conceptual Modeling", (2005) October 24-28; Klagenfurt, Austria

[13] F. Yang and B. L, Yin, "Journal of Southeast University", 23, 3(2007)

[14] Y. Z. Zhu and B. L. Yin, "Journal of Computational Information Systems", 9, 20(2013)

[15] Y. Z. Zhu, H. Yang and B. L. Yin, "Computer Modeling and New Technologies", 18, 1(2014)

[16] W. M. P. van Der Aalst, A. H. M. Ter Hofstede, B. Kiepuszewski and A. P. Barros, "Distributed and parallel Databases", 14, 1(2003) 


\section{Authors}

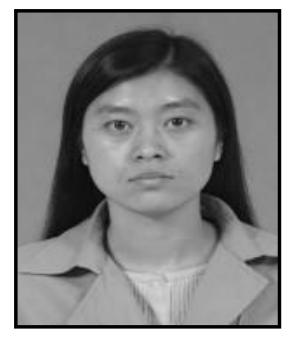

F. Yang, received her $\mathrm{PhD}$ in Computer Sciences and Engineering Department(2011) from Beihang University. Now she is full lecturer at Computer Department, Beijing Institute Petrochemical Technology. Her current research interests include workflow management, enterprise application integration and distributed system.

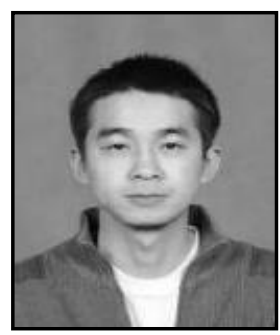

Y.Z. Zhu, is a Ph.D. candidate in Computer Sciences and Engineering Department at Beihang University.

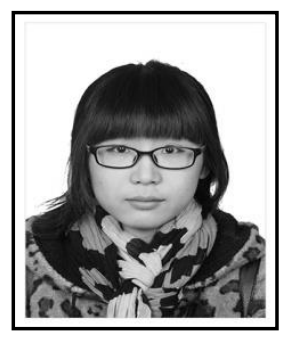

Y. Luo, is an undergraduate student in Computer Sciences and Engineering Department from Beijing Institute Petrochemical Technology. 
International Journal of Control and Automation Vol.8, No.10 (2015) 